\title{
Development of WDM System in Optical Amplifiers by Manipulating Fiber Length and Bandwidth for Telecommunication System
}

\author{
Roby Ikhsan ${ }^{1}$, Romi F. Syahputra ${ }^{1}$, Suhardi $^{1}$, Saktioto $^{1(凶)}$, \\ Nor Ain Husein ${ }^{2}$, and Okfalisa ${ }^{3}$ \\ ${ }^{1}$ Department of Physics, Universitas Riau, Jl. HR Soebrantas KM. 12, 5, \\ Pekanbaru 28293, Riau, Indonesia \\ ikhsanroby7@gmail.com, romifadlisyahputra@yahoo.com, \\ salimihamzah@gmail.com, saktioto@yahoo.com \\ 2 Jabatan Fizik, Fakulti Sains, Universiti Teknologi Malaysia, \\ 81310 Skudai, Johor, Malaysia \\ norainh@utm.my \\ ${ }^{3}$ UIN Sultan Syarif Kasim, Jl. HR Soebrantas KM. 15, \\ Pekanbaru 28293, Riau, Indonesia \\ okfalisa@gmail.com
}

\begin{abstract}
The use of fiber optic for telecommunication system has widely developed because of limitations on communication ranges and methods for information demands. In the application of optical fiber communications, signal strength is reduced due to attenuation, absorption and dispersion effects of source, structure, and geometry of fiber, even for single mode fiber, these problems still exist. In order to investigate these phenomena, this paper designs and operates a simple optical design consisting of wavelength division multiplexing (WDM) which is able to multiplex various wavelength sources to one fiber optic by using various source wavelengths. This mechanism allows a bidirectional communication with several or more fibers without any interference. The output will describe the bit error rate (BER) and Q-factor and using two samples of Semiconductor Optical Amplifier (SOA) and Fiber Raman Amplifier (FRA). The simulation evaluates these amplifiers by manipulating the frequency of bandwidth until $50 \mathrm{GHz}$, a low BER corresponding to large Q-factor is reached. The BER value for SOA device is $7.59 \times 10^{-16}$ and $1.54 \times 10^{-27}$ for SOA device respectively for a bandwidth of $50 \mathrm{GHz}$. These data depict that SOA is able to launch the wavelength to high performance than that of FRA device. Both optical amplifiers describe better performances in term of the value of BER and Q-factor having much smaller than $10^{-12}$ and larger than that of 6 respectively for a distance of $120 \mathrm{~km}$ for SOA and $100 \mathrm{~km}$ for FRA.
\end{abstract}

Keywords: Optical fiber $\cdot$ Optical circuit $\cdot$ WDM $\cdot$ FRA $\cdot$ SOA 


\section{Introduction}

A high speed telecommunication system is a key success in transferring information around the world, whether the space is separated by miles away. One of the information carriers is an electromagnetic wave which has various frequencies. Electromagnetic wave applied in optical circuit and communication systems uses high carrier frequencies source $(\sim 100 \mathrm{Tbit} / \mathrm{s})$ in the visible or near-infrared wavelength region [1]. Those sources of carrier frequency bandwidth are particularly much smaller by five orders of the value $(\sim 1 \mathrm{GHz})$ [2]. Optical fiber circuit and communication system are able to deal with optical fiber as media for carrying, transferring and filtering data and information from one place to another. Optical fiber system generally consists of transmitting, receiving and filtering devices which are able to convert electrical to the light signal. This fiber cable also carries the visible and invisible light and a receiver device which admits the spectral signal back to change into an electrical signal.

There has been a massive need to advance the communication system with the swift growth in telecommunication systems such as fast-signal internet device and error linkage system which meet the first need and commit for the service in term of a provider [3, 4]. These service and development have produced an increased high concern in applying a wavelength division multiplexing (WDM) for advanced optical communication and network system.

WDM component has the function and capability of transmitting into a single fiber optics and multiple numbers of waves with various frequencies source. Kinds of data and information channels in term of wavelength and intensity can be added to a different location by applying an add/drop multiplexer system. That adding system can improve and fit the flexibility of the WDM system [4-6]. Sending multiple signals is needed to gain a high bandwidth in optical communication. WDM systems come to fulfill this necessity. However, this communication still faces a lot of obstacle such as wavelength and refractive indices resulting a dispersion problem [5-8]. Dispersion results in the widening signal and influences the source wavelength so that the intensity losses will be obtained from the original data at the receiver end.

There are a lot of techniques which have developed to play as dispersion compensation such as using FBG [7-11], chirp grating [12], artificial neural network [13], optoelectronic method [14], dispersion compensate fiber [15], and orthogonal frequency division multiplexing [16]. Another technique is using an optical amplifier. Optical amplifier are widely used and become component in telecommunication networks. These are hoped to have a better service and maintenance towards all system of optical circuit by applying optical amplifiers corresponding to another optical component, and of course, this requires efficient and effective optical amplifiers because of large power losses as far as the optical circuit operates. This circuit and link suffer from attenuation and dispersion. The attenuation from geometry effect produces intensity signal losses and restricts the transmission ranges. Nevertheless, the beam source input will broaden as a result of the dispersion. It will lead to interference, limitation in the fiber signal and increase bit error rate (BER) [17]. Here, an optical amplifier is required to improve and compensate for the intensity losses. Nowadays, kinds of optical amplifier technologies that have developed with distinguished properties. SOA has 
been used to compensate dispersion $[18,19]$. In this paper, two amplifiers are chosen to investigate the performance of amplification ranges i.e., SOA and FRA. They will be compared at various bandwidth and length of the fiber. The other one, Erbium-Doped is not considered in this result, where it is underway to model for different purposes.

\section{Design and Operation}

The circuit is dealt with basic optical communication having components which consist of transmission link, filter, transmitter, and a receiver device [20]. A design is set by preparing the power input and several optical components to deliver, change and increase the signal where it is found reduced. The system launches the power bringing data and information and transmits it using electromagnetic wave from the source to the receiver through optical fiber as shown in Fig. 1. The optical circuit contains four transmitters which use equal channel spacing of $50 \mathrm{GHz}$. This circuit is operated by simulating the power input and output. The design and operation for simulation are described as follows.

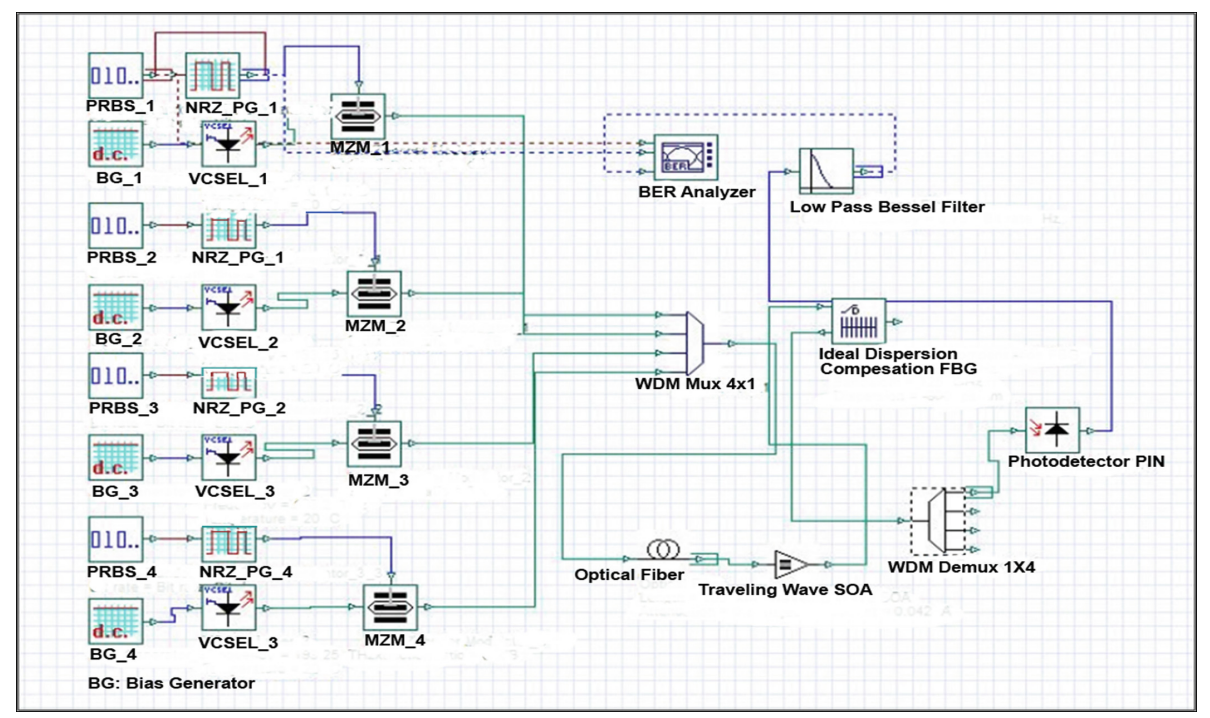

Fig. 1. WDM system using SOA

The electromagnetic input containing electrical signal data is shown by 0's and 1's generated by a non-return-zero (NRZ) pseudo-random binary sequence (PRBS) [21]. This signal source will be then modulated with a visible or invisible laser source generated by Vertical Cavity Surface Emitting Laser (VCSEL) through Mach-Zehnder Modulator (MZM). VCSEL's supply input signal starting from $193.1 \mathrm{THz}$ to 193.25 THz of frequency and temperature of $20{ }^{\circ} \mathrm{C}$. MZM will mix the input of electrical signal and produce optical signal as the output which delivered into the multiplexer. 
Single mode fiber is applied for fewer intensity losses. It can also result greater data transfer with low dispersion and enable to operate in long optical fiber system. Therefore it is compatible to deal with a transmission link. This transmission link circuit using SMF, the operation circuit system results in a dispersion of about $16.75 \mathrm{ps} / \mathrm{km} / \mathrm{nm}$ and attenuation of $0.3 \mathrm{~dB} / \mathrm{km}$. The $50 \mathrm{~km}$ of fiber length draw on various bandwidth simulations.

This simulation uses FBG functioning the wavelength filter and transmission as dispersion compensation and amplifier as fiber linear loss compensation (attenuation). The optical circuit system is set for $50 \mathrm{~km}$ fiber optics length which is combined with SOA by injecting electrical current of $0.042 \mathrm{~A}$, and followed by the attenuation of the fiber is $0.3 \mathrm{~dB} / \mathrm{km}$. The four-channel frequencies are $193.1 \mathrm{THz}, 193.15 \mathrm{THz}$, 193.2 THz and 193.25 THz as shown in Fig. 1. Raman Amplification specifies a length of $10 \mathrm{~km}$ and pumps laser frequency of $980 \mathrm{~nm}$ as depicted in Fig. 2.

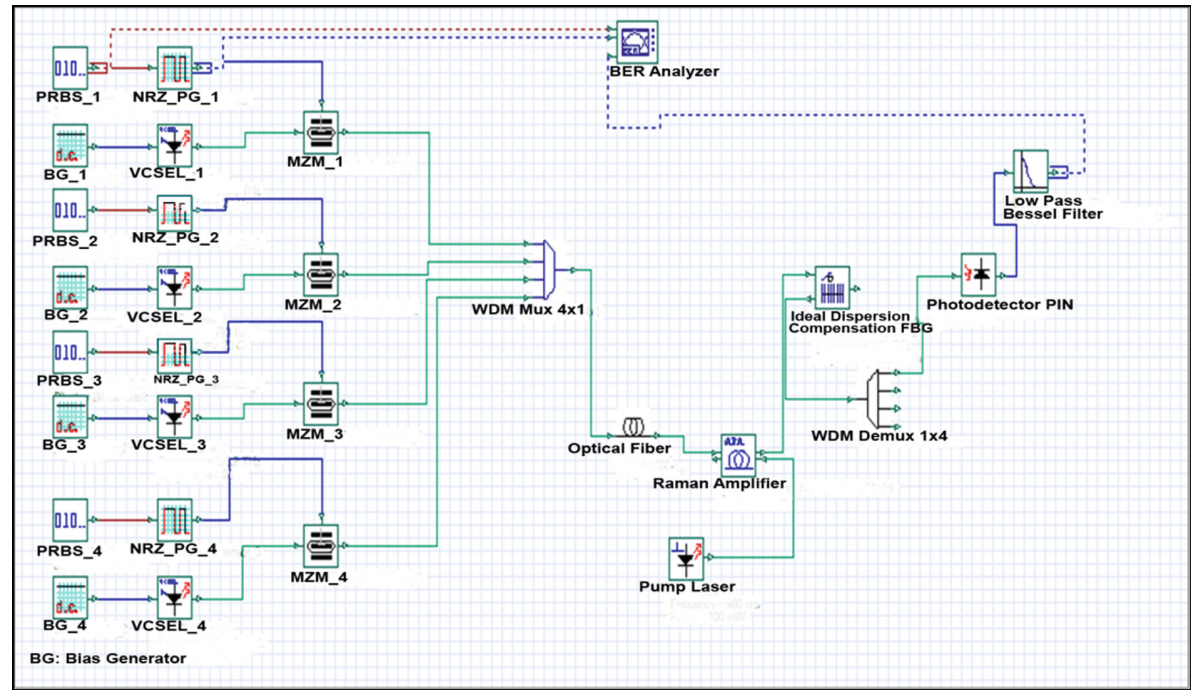

Fig. 2. WDM system using FRA.

For block receiver component, PIN photo-detector is set as a sensor of the optical signal and change the wavelength source to electrical signal and deliver it to low pass filter by using Bessel filter function which is filtering high frequency carrier signal and transmitting the low frequency signal The BER analyzer is then applied to summarize BER and quality factor (Q-factor) of the optical design and operation circuit system.

\section{Result and Discussion}

The optical circuit proposed in Figs. 1 and 2 are design and operation of the amplifier system having a frequency of $10 \mathrm{GHz}$ bandwidth and they produce the output results for the Q-factor is 5.44 for SOA and 7.07 for FRA respectively. These outputs are also detected by eye diagrams as shown in Fig. 3. 

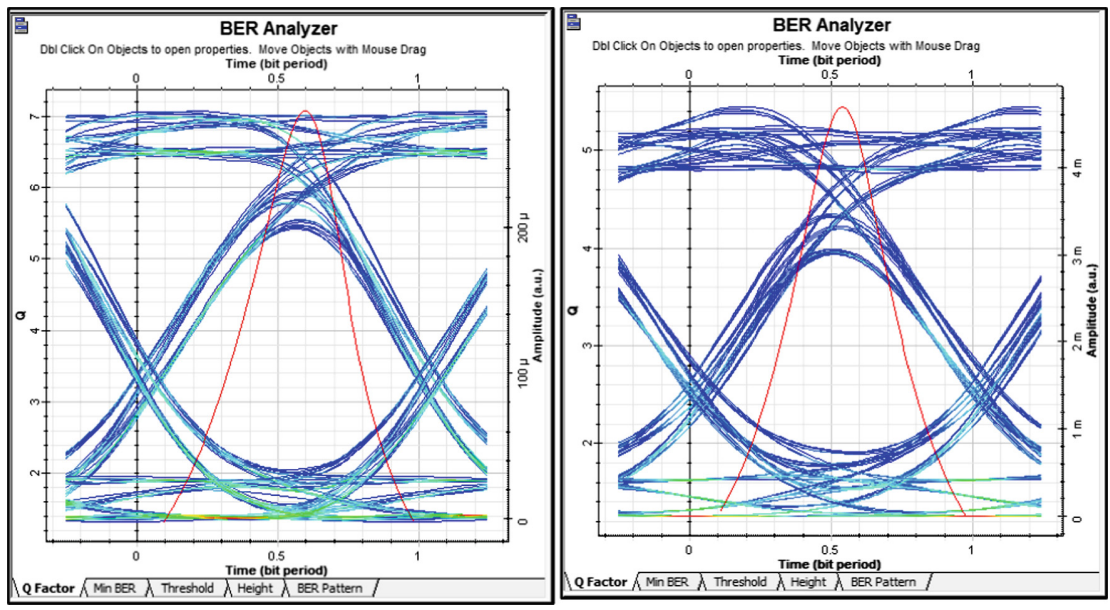

Fig. 3. Eye diagram of FRA (left) and SOA (right) for $10 \mathrm{GHz}$ bandwidth

The increment of the bandwidth until $20 \mathrm{GHz}$ will also produce the Q-factor increased, and BER is decreased and of course, it is required for optical transmission. However, when the bandwidth is higher than $20 \mathrm{GHz}$, the Q-factor and BER tend to be stable. Furthermore, BER, Q-factor value and the eye diagrams for various values is shown in Fig. 4. Figure 4 describes the eye diagrams look slightly smooth although the $10 \mathrm{Ghz}$ is better than $20 \mathrm{GHz}$. The small slope of the eye diagram indicates that the jitter is not significant effect. The value of noise which can be tolerated is rather high, it means the small value of noise occurs hence the small distortion occurs [22]. The formed eye diagram is not surprising where the losses occur, much power is wasted and the value of noise allowed is still small. This shows that the gradient resulted produces the sensitivity of the jitter is large.
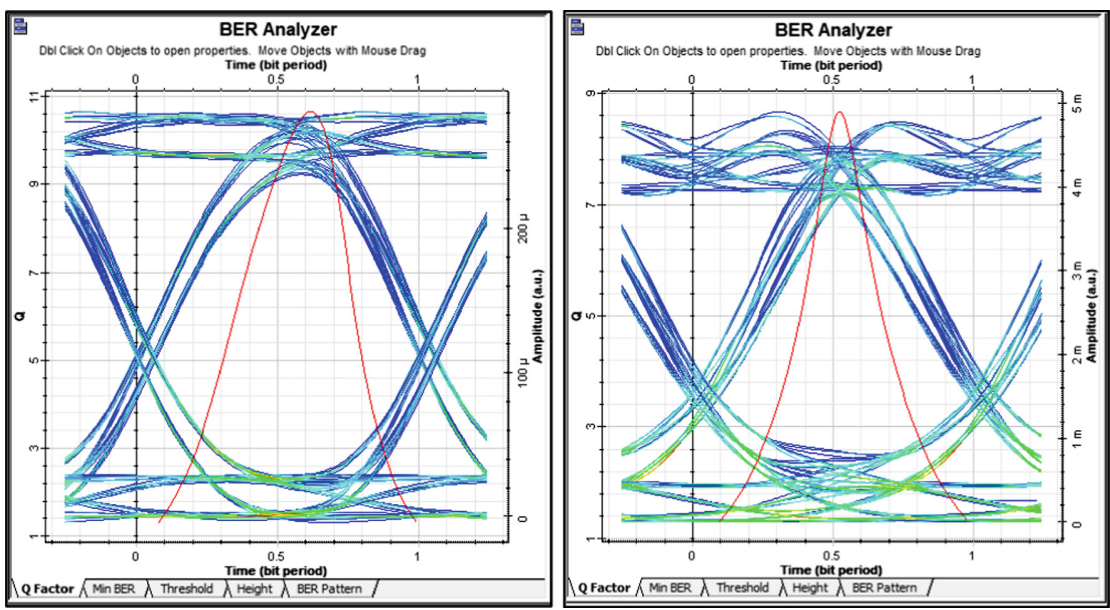

Fig. 4. Eye diagram of FRA (left) and SOA (right) for $20 \mathrm{GHz}$ bandwidth 
These eye diagrams can produce interferences by effects of multipath where the signal wave from the input source, mainly as power is delivered to a sensor by two or more paths, with certain condition, the components of the electromagnetic wave can interfere. The wave signal propagates where the modulation is formed by the various signal patterns such as sine and cosine inputs at a precise function of position and time. Both diagrams are geometrically a better performance even though it seems that there is a slight distortion because of dispersion condition as depicted at the certain lines shown by 'left arm' and 'right arm' in the picture. Of course, there is also noise occurs such as top line (noise "1") compared to down line (noise " 0 ").

As shown in the eye diagram, the opening of eye as it is set at the middle region, the performance of FRA is large compared to SOA device at set at the center. The number of disturbance can be allowed by the wave described as a vertical length of the diagram is high and also a horizontal length of the diagram is large. The length of horizon describing the time function to the waveform informs that the sensitivity to timing error is small, meaning the pattern is good. The time jitter is also tiny as described by the bit period of value of 1 and 0 . In conclusion, in accordance with the graph and geometry analysis, FRA is better result than that of SOA.

From Table 1, it can be observed both SOA and FRA when the increment of bandwidth, the value of Q-factor goes up but otherwise bit error rate goes down. If there is no SOA component, then Q-factor is zero and BER is 1. More details information of Q-factor is depicted in Fig. 5.

Table 1. Q-factor and BER by varying the bandwidth applying SOA and FRA

\begin{tabular}{l|l|r|l|l|l}
\hline \multirow{2}{*}{ No } & \multirow{2}{*}{ Bandwidth (GHz) } & Q-Factor & \multicolumn{2}{l}{ BER } \\
\cline { 3 - 6 } & & FRA & SOA & FRA & SOA \\
\hline 1 & 10 & 7.07 & 5.44 & $7.26 \times 10^{-13}$ & $2.45 \times 10^{-08}$ \\
\hline 2 & 20 & 10.65 & 8.66 & $8.60 \times 10^{-27}$ & $2.25 \times 10^{-18}$ \\
\hline 3 & 30 & 11.16 & 8.48 & $3.16 \times 10^{-29}$ & $1.12 \times 10^{-17}$ \\
\hline 4 & 40 & 11.05 & 8.63 & $1.04 \times 10^{-28}$ & $3.02 \times 10^{-18}$ \\
\hline 5 & 50 & 10.81 & 7.98 & $1.54 \times 10^{-27}$ & $7.59 \times 10^{-16}$ \\
\hline
\end{tabular}

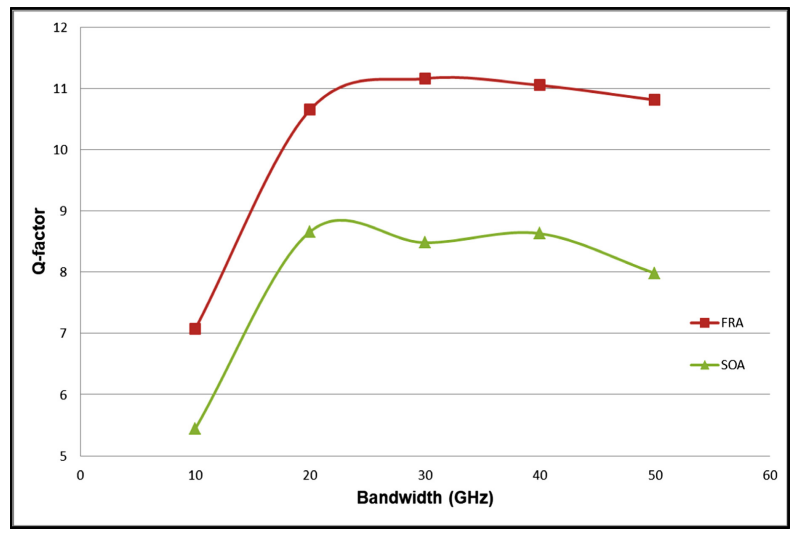

Fig. 5. Q-factor for FRA and SOA 
As seen in Table 2, it is found that when the increment of fiber length until $40 \mathrm{~km}$, the value of Q-factor will be high but opposite to BER. However, when fiber length is above $40 \mathrm{~km}$, it will be vice versa for amplifier quality. The Q-factor is also shown in Fig. 6. The propagation of signals due to the higher frequency used to result in the quality of wave reduced corresponding to energy decreased. This also occurs when the length of fiber is long [23], then the power losses are affected by the geometry and structure of fibers which imply to the SOA and FRA. As depicted in Fig. 6, FRA quality factor goes down if the fiber length increases. It is not surprised since the FRA is much affected by the frequency then the power is much lower [24]. However, this does not explain that FRA performance is not good enough since the wave propagation is not readily reached due to attenuation.

Table 2. Optical amplifier quality for SOA and FRA

\begin{tabular}{l|l|l|l|l|l}
\hline No & Fiber length & Q-Factor & \multicolumn{2}{l}{ BER } \\
\cline { 2 - 5 } & $(\mathrm{km})$ & FRA & SOA & FRA & SOA \\
\hline 1 & 20 & 9.29 & 7.83 & $7.23 \times 10^{-21}$ & $2.51 \times 10^{-15}$ \\
\hline 2 & 40 & 13.43 & 9.42 & $2.08 \times 10^{-41}$ & $2.05 \times 10^{-21}$ \\
\hline 3 & 60 & 9.7 & 7.79 & $1.52 \times 10^{-22}$ & $3.28 \times 10^{-15}$ \\
\hline 4 & 80 & 8.15 & 7.97 & $1.78 \times 10^{-16}$ & $7.76 \times 10^{-16}$ \\
\hline 5 & 100 & 6.05 & 8.24 & $7.30 \times 10^{-10}$ & $8.87 \times 10^{-17}$ \\
\hline 6 & 120 & 2.96 & 9.23 & $1.52 \times 10^{-03}$ & $1.03 \times 10^{-20}$ \\
\hline 7 & 140 & 0 & 7.86 & 1 & $1.96 \times 10^{-15}$ \\
\hline
\end{tabular}

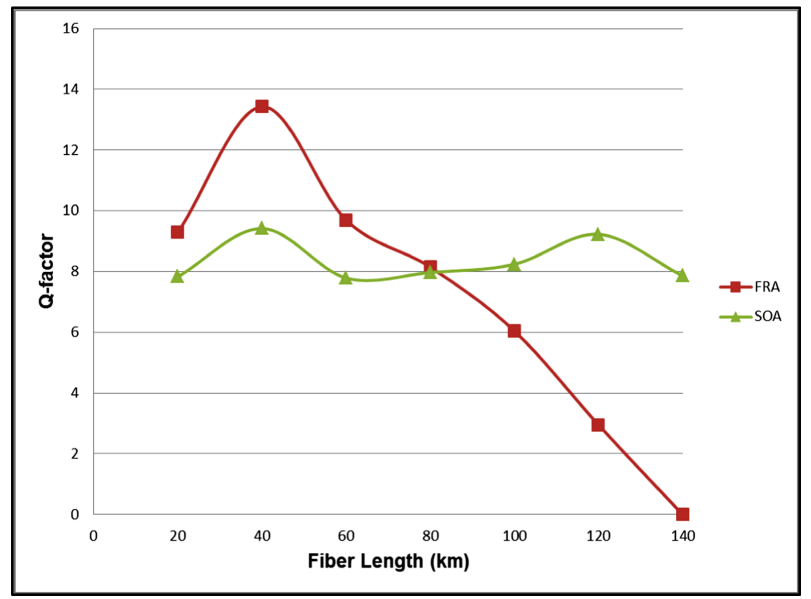

Fig. 6. Q-factor for various fiber length 


\section{Conclusion}

This article can be concluded that the WDM system for SOA and FRA has been successfully designed, operated and compared. The optical circuit is set for $50 \mathrm{~km}$ length injecting electrical current of $0.042 \mathrm{~A}$ for SOA and is found that the intensity loss of the optical fiber is about $0.3 \mathrm{~dB} / \mathrm{km}$. The four-channel frequencies are set on the above $\mathrm{THz}$ regions which relate to channel spacing of $50 \mathrm{GHz}$. For $20 \mathrm{GHz}$ bandwidth, it is obtained that the BER is low and Q-factor is high. Beyond the signal frequency of $20 \mathrm{GHz}$, Q-factor and BER value have nearly linear profile. Also, based on geometry analysis of eye diagram, FRA device has a better performance compared to SOA device having the highest Q-factor and lowest BER value. Nonetheless, FRA device is actually not as stable as the SOA device.

\section{References}

1. Agrell, E., Karlsson, M., Chraplyvy, A.R., Richardson, D.J., Krummrich, P.M., Winzer, P., et al.: Roadmap of optical communications. J. Opt. 18(6), 063002 (2016)

2. Agrawal, G.P.: Fiber-Optic Communication Systems, 4th edn. Wiley, New York (2010)

3. Panda, T.K., Mishra, P., Patra K.C., Barapanda, N.K.: Investigation and performance analysis of WDM System implementing FBG at different grating length and datarate for long haul optical communication. In: 2017 IEEE International Conference on Power, Control, Signals and Instrumentation Engineering (ICPCSI), pp. 483-488. IEEE New York (2017)

4. Collings, B.C., Mitchell, M.L., Boivin, L., Knox, W.H.: A 1021 channel WDM system. IEEE Photonics Technol. Lett. 12(7), 906-908 (2000)

5. Keiser, E.G.: A review of WDM technology and applications. Optical Fiber Technology 5 (1), 3-39 (1999)

6. McCoy, A.D., Thomsen, B.C., Ibsen, M., Richardson, D.J.: Filtering effects in a spectrumsliced WDM system using SOA-based noise reduction. IEEE Photonics Technol. Lett. 16(2), 680-682 (2004)

7. Othman, M.A., Ismail, M.M., Sulaiman, H.A., Misran, M.H., Said, M.A.M., Rahim, Y.A., ChePee, A.N., Motsidi, M.R.: An analysis of $10 \mathrm{Gbits} / \mathrm{s}$ optical transmission system using fiber Bragg grating (FBG). IOSR J. Eng. (IOSRJEN) 2(7), 55-61 (2012)

8. Prashad, B., Mallick, B., Parida, A.K.: Fiber Bragg grating as a dispersion compensator in an optical transmission system using optisystem software. Int. Res. J. Eng. Technol. (IRJET) 2 (6), 9-14 (2014)

9. Kumar, K., Jaiswal, A.K., Kumar, M., Agrawal, N.: Performance analysis of dispersion compensation using fiber Bragg grating (FBG) in optical communication. Int. J. Curr. Eng. Technol. 4(3), 1527-1531 (2014)

10. Litchinitser, N.M., Patterson, D.B.: Analysis of fiber Bragg gratings for dispersion compensation in reflective and transmissive geometries. J. Lightwave Technol. 15(8), 1323-1328 (1997)

11. Sharma, A., Singh, S., Sharma, B.: Investigations on dispersion compensation using fiber Braggs grating. Int. J. Comput. Appl. 73(2), 34-43 (2013)

12. Mohammadi, S.O., Mozzaffari, S., Shahidi, M.M.: Simulation of a transmission system to compensate dispersion in an optical fiber by chirp gratings. Int. J. Phys. Sci. 6(32), 7354$7360(2011)$ 
13. Warm, S., Bunge, C.-A., Wuth, T., Petermann, K.: Electronic dispersion precompensation with a $10 \mathrm{~Gb} / \mathrm{s}$ directly modulated laser. IEEE Photonics Technol. Lett. 21(15), 1090-1092 (2009)

14. Foo, B., Corcoran, B.: Lowery, a: optoelectronic method for inline compensation of XPM in long-haul optical links. Opt. Express 23(2), 859-872 (2015)

15. Nielsen, L.G., Wandel, M., Kristensen, P., Jorgensen, C., Jorgensen, L.V., Edvold, B., Palsdottir, B., Jakobsen, D.: Dispersion-compensating fibers. J. Lightwave Technol. 23(11), 3566-3579 (2005)

16. Lowery, A.J., Armstrong, J.: Orthogonal-frequency-division multiplexing for dispersion compensation of long-haul optical systems. Opt. Express 14(6), 2079-2084 (2006)

17. Golani, O., Elson, D., Lavery, D., Galdino, L., Killey, R., Bayvel, P., Shtaif, M.: Experimental characterization of nonlinear interference noise as a process of intersymbol interference. Opt. Lett. 43(5), 1123-1126 (2018)

18. Hossain, M.S., Howlader, S., Basak, R.: Investigating the Q-factor and BER of a WDM system in optical fiber communication network by using SOA. Int. J. Innov. Sci. Res. 13(1), 315-322 (2015)

19. Vedala, G., Hameed, M.A., RongqingHui, R.: Digital compensation of SSBI in direct detection multicarrier system with SOA nonlinearities. IEEE Photonics Technol. Lett. 29(4), 369-372 (2017)

20. Kikuchi, K.: Fundamentals of coherent optical fiber communications. J. Lightwave Technol. 34(1), 157-179 (2016)

21. Aldouri, M.Y., Aljunid, S.A., Ahmad, R.B., Fadhil, H.A.: Bit error rate (BER) performance of return-to-zero and non-return-to-zero data signals optical code division multiple access (OCDMA) system based on and detection scheme in fiber-to-the-home (FTTH) networks. Opt. Appl. 41(1), 173-181 (2011)

22. Kim, J.-H., Lim, J.-H., Kim, B., Sim, J.-Y., Park, H.-J.: An adaptive equalizer for high-speed receiver using a CDR-assisted all-digital jitter measurement. J. Semicond. Technol. Sci. 15 (2), 155-167 (2015)

23. Marhic, M.E., Andrekson, P.A., Petropoulos, P., Radic, S., Peucheret, C., Jazayerifar, M.: Fiber optical parametric amplifiers in optical communication systems. Laser Photonics Rev. 9(1), 50-74 (2015)

24. Clivati, C., Bolognini, G., Calonico, D., Faralli, S., Mura, A., Levi, F.: In-field Raman amplification on coherent optical fiber links for frequency metrology. Opt. Express 23(8), 10604-10615 (2015) 